\title{
A Determination of the Wave Forms and Laws of Propagation and Dissipation of Ballistic Shock Waves*
}

\author{
Jesse W. M. DuMond, E. Richard Cohen, W. K. H. Panofsky, and Edward Deeds \\ California Institute of Technology, Pasadena, California
}

(Received September 27, 1945)

\begin{abstract}
Experiments to ascertain the wave forms and laws of propagation and dissipation of ballistic shock waves to large distances $(80$ yards) from the bullet trajectory are described. Calibers $0.30,0.50,20 \mathrm{~mm}$, and $40 \mathrm{~mm}$ were studied. In every case an $\mathrm{N}$-shaped wave profile was observed consisting of a sudden rise in pressure, the "head discontinuity," followed by an approximately linear decline to a pressure about equally far below atmospheric and then a second sudden return, the "tail discontinuity," to atmospheric pressure. The peak amplitudes of this disturbance are found to diminish about as the inverse $\frac{3}{4}$ power of the miss-distance (perpendicular distance from the trajectory) while the period $T^{\prime}$ (measured between the discontinuous fronts) increases about as the $\frac{1}{4}$ power of the miss-distance for calibers 0.30 , 0.50 , and $20 \mathrm{~mm}$. For $40-\mathrm{mm}$ shells the amplitude decays a little faster, about as the inverse 0.9 power of miss-distance over the range studied. A theory taking account of the dissipation of the $\mathrm{N}$-wave energy into heat is developed to explain the observed behavior. A method of measuring absolute $\mathrm{N}$-wave amplitudes by observing the rate of change of period $T^{\prime}$ with propagation is described. The theory leads to an absolute relationship at large distances between distance, amplitude, and period in which no arbitrary constants appear.
\end{abstract}

\section{A. EXPERIMENTAL OBSERVATIONS}

\section{Objectives and Field Measurements}

$\mathrm{F}$ Rertain military applications it has become desirable to understand the nature, wave forms, and laws of propagation of the ballistic shock waves emanating from the trajecjectories of bullets whose velocities are considerably in excess of the velocity of sound. Information regarding the nature of the shock waves and their attenuation at rather large distances from the trajectory, indeed as far away as 70 or 80 yards, was desired. Because of the difficult nature of the measurements, subject as they are to several sources of considerable statistical fluctuation, it was highly desirable to develop a valid theoretical framework into which experimental results could be fitted.

An extensive program of field measurements with several types of microphone was undertaken. We summarize these studies as follows:

1. Calibers studied: Caliber $0.30,0.50,20 \mathrm{~mm}, 40 \mathrm{~mm}$.

2. Miss-distances (perpendicular distance from trajectory to microphone): 0 to 80 yards.

3. Microphones used: quartz piezoelectric (Bell Telephone Laboratories) with frequency-flat pressure calibration to about 100,000 c.p.s.; specially developed condenser

* This paper is based on work done for the Office of Scientific Research and Development under Contract No. OEMsr-600 with the California Institute of Technology. microphones with frequency-flat pressure calibration to 10,000 c.p.s.; high " $Q$ " or resonant diaphragm condenser microphones having a " $Q$ " from 50 to 100 and natural frequencies of 1600 and of 2400 c.p.s.

\section{Recording Equipment}

A single sweep oscilloscope (with persistent screen and spot intensification) triggered by a microphone was placed a few feet in advance of the recording microphone. The oscilloscope records were photographed.

Peak amplitudes of the condenser microphone responses were also recorded by equipment in which the output microphone signals were electronically pulse-lengthened and suitably amplified so as to deflect the pen of one or more Esterline-Angus recording milliammeters.

\section{B. RESULTS OF FIELD MEASUREMENTS}

\section{Wave Forms of Ballistic Shock Waves}

The same general wave form was found to characterize the ballistic shock waves from all

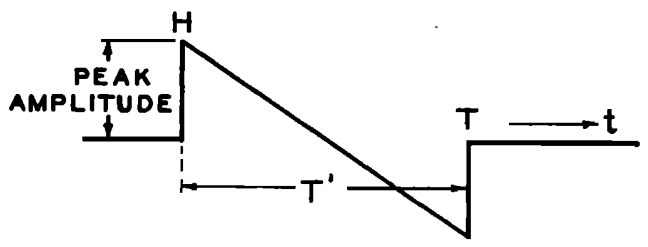

FIG. 1. Pressure profile of $\mathrm{N}$-wave as a function of the time. 
the calibers studied. This consists of a very sudden rise in pressure followed by a nearly linear pressure decline to a value about as far below atmospheric as the original rise and then a very sudden return to atmospheric pressure. Figure 1 shows the profile we are describing which because of its shape we shall frequently refer to as an "N-wave." 1 The two sudden rises in pressure (which we shall frequently refer to as "discontinuities") are separated by a time interval $T^{\prime}$ which gradually increases as the $\mathrm{N}$ wave is propagated. This time interval we shall refer to as the shock wave "period." We have found empirically that $T^{\prime}$ is approximately proportional to the fourth root of the miss-distance. $T^{\prime}$ increases with increasing caliber. Figure 2 gives on logarithmic scale the results of our measurements of $T^{\prime}$ for different calibers and miss-distances.

As we shall soon see, this increase in the shock wave period $T^{\prime}$ with propagation is caused by the fact that the two discontinuous fronts $H$ and $T$ in the wave form are propagated at velocities, respectively, higher and lower than ordinary sonic velocity. These differences from sonic velocity can be definitely related to the peak amplitudes of the pressure steps $H$ and $T$ by a formula" frequently called the "RankineHugoniot discontinuity_relationship," a simple

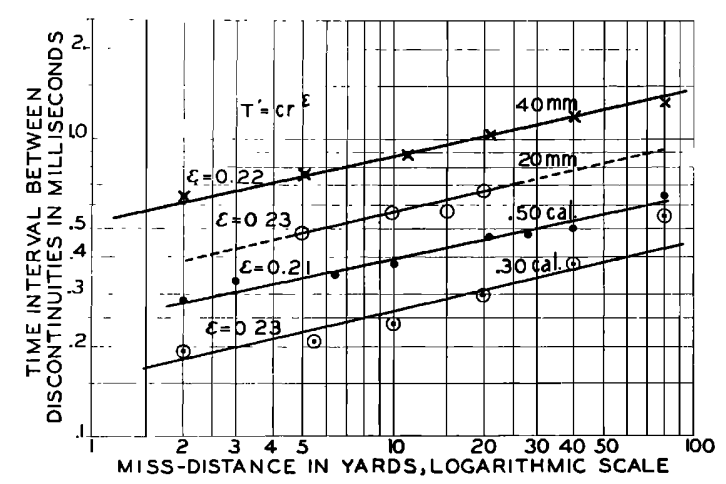

FIG. 2. Period of shock wave $v s$. miss-distance.

${ }^{1} \mathrm{~L}$. D. Landau in a paper briefly abstracted in J. Phys. Acad. Sci. U.S.S.R. 6, 229-230 (1942) describes a wave form of this type which theoretical considerations lead him to expect as the eventual profile of a shock wave at sufficient distance from the source, a supersonic projectile or a central explosion. The abstract contains equations equivalent to our Eqs. (7.1) and (8.1), Section IV, part D, and Eqs. (7) and (8), Section V, part D; the proof is not included. expression of which we develop in a later section (Section D I, Eq. (10)). This fact makes it possible to measure the peak pressure amplitudes of $\mathrm{N}$-waves by the rate of change of $T^{\prime}$ with miss-distance and this method though too cumbersome for most practical applied measurements possesses the advantage for an absolute determination of amplitude that it is not subject to the uncertainties of direct pressure measurements made with microphones (because of the reflected wave, and the diffraction of sound around the microphone case, etc.), since it involves essentially only the measurement of time intervals. The chief difficulty in its application arises for the smaller calibers where relatively large superposed accidental disturbances on the oscillograms may make it difficult to identify the $H$ and $T$ discontinuities with certainty. It will appear later that the method is also restricted to a region of miss-distances which for best reliability must be neither too close nor too distant from the trajectory.

The discontinuities $H$ and $T$ of the $\mathrm{N}$-wave can be seen in direct photographs of ballistic shock waves as the two sharp lines trailing backward, respectively, from the nose and tail of the bullet. (See Fig. 5 below.)

\section{Peak Amplitudes of Ballistic Shock Waves}

Our measurements have shown peak amplitudes of ballistic shock waves (the amplitude of the pressure step, $H$ or $T$ ) to depend on missdistance, $d$, according to the law

$$
\begin{aligned}
& \text { Amplitude }=K / d^{n} ; K=\text { a constant depending } \\
& \text { on caliber. } \frac{3}{4}<n<1 .
\end{aligned}
$$

The exponent $n$ appeared to have about the value $\frac{3}{4}$ for the three smaller calibers studied but for $40-\mathrm{mm}$ bullets $n$ was more nearly equal to unity (about $0.9 \pm 0.1$ ). From the theory developed in this paper it appears that $n$ should be weakly dependent on the amplitude of the shock wave itself (and should therefore vary slowly with miss-distance) but should in no case be numerically less than $\frac{3}{4}$. The statistical fluctuations in the amplitude measurements with microphones made it difficult by such means to determine $n$ very precisely and impossible to detect changes in $n$ with miss-distance for any given 


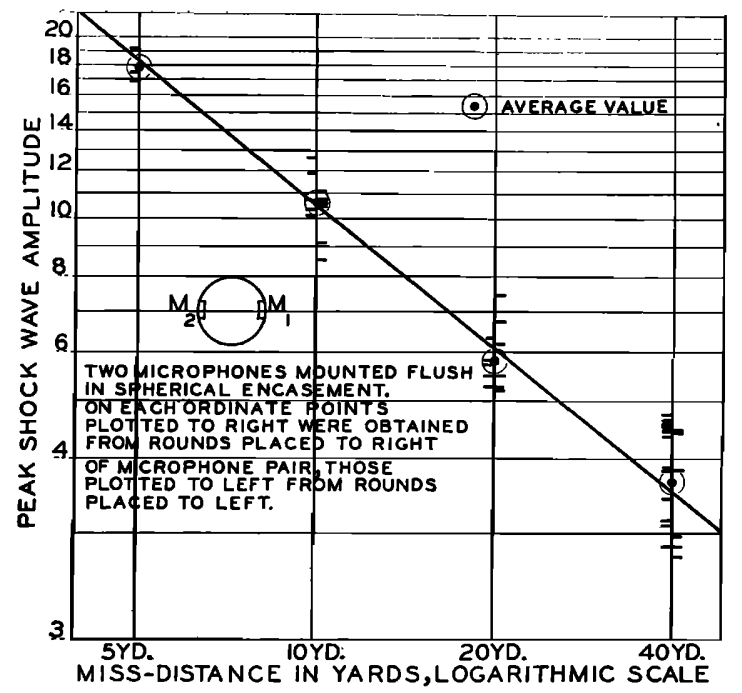

FIG. 3. Experimental data for .50 caliber. Transmitter No. 8;

Range: 500 yards;

Location: Camp Irwin;

Date: November 8, 1944;

Round number : $85 \mathrm{~A}-87 \mathrm{Z}$.

caliber. Figures 3 and 4 show logarithmically our experimental observations as to the dependence of amplitude on miss-distance for two calibers. The measurements were made with two microphones mounted at diametrically opposite points of a $7 \frac{3}{4}$-inch rigid spherical case, the diaphragms being nearly flush with the surface of the sphere. It can be shown both theoretically and experimentally that the sum of the responses of the two microphones should, with this arrangement, be nearly independent of the orientation of the sphere. Shots were placed on either side to right or to left of the microphone pair axis, and the sum of the two responses thus obtained is plotted in the diagram just to right or to left of the miss-distance ordinate.

Referring to Fig. 5 the shock wave energy from a segment $O O^{\prime}$ of bullet trajectory flows outward, remaining essentially between the two conical boundaries $O P$ and $O^{\prime} P^{\prime}$, to the shaded region at average miss-distance $d$ as shown. At the instant shown the energy from $O O^{\prime}$ fills the volume of revolution having the trajectory as axis of revolution and the section shown by the shading in the figure. The inner and outer boundaries of this section are the $H$ and $T$ discontinuities. If these discontinuities were not

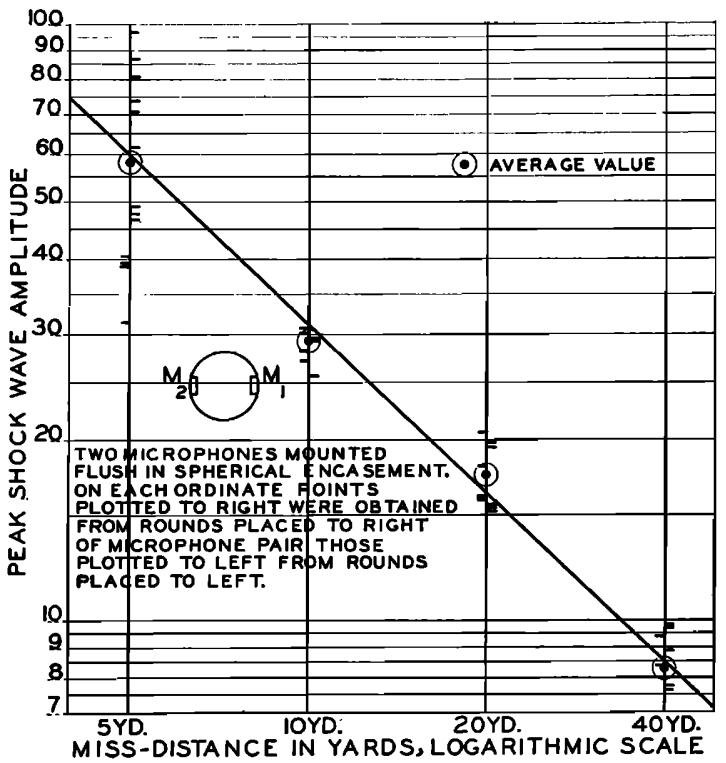

FIG. 4. Experimental data for $40 \mathrm{~mm}$.

Transmitter 10;

Range: 500 yards;

Location: Camp Irwin

Date: November 9, 1944;

Round number: $90 \mathrm{~A}-91 \mathrm{~V}$.

gradually spreading apart the volume enclosing the wave energy in question would increase in direct proportion to the miss-distance $d$. If this wave energy remained constant without dissipation, one would therefore expect the intensity of the ballistic shock wave to vary inversely as the miss-distance and the amplitude to vary inversely as the square root of miss-distance.

It can be shown by calculation that the observed faster rate of decay of $\mathrm{N}$-wave amplitude with miss-distance (the $-\frac{3}{4}$ power instead of $-\frac{1}{2}$ power) cannot be accounted for by the spreading apart of the $H$ and $T$ discontinuities alone (i.e., because of the resulting faster increase in the volume occupied by the wave energy). Such a calculation makes it clear that to account for the $-\frac{3}{4}$ power law of amplitude decay it is very definitely necessary to take into account also the dissipation of the $N$-wave energy (its degradation into heat) as the wave propagates. It is the purpose of this paper to develop a theory taking all these factors into account and to show that such a theory is consistent with the measurements from field observations. Certain new and interesting absolute relations be- 


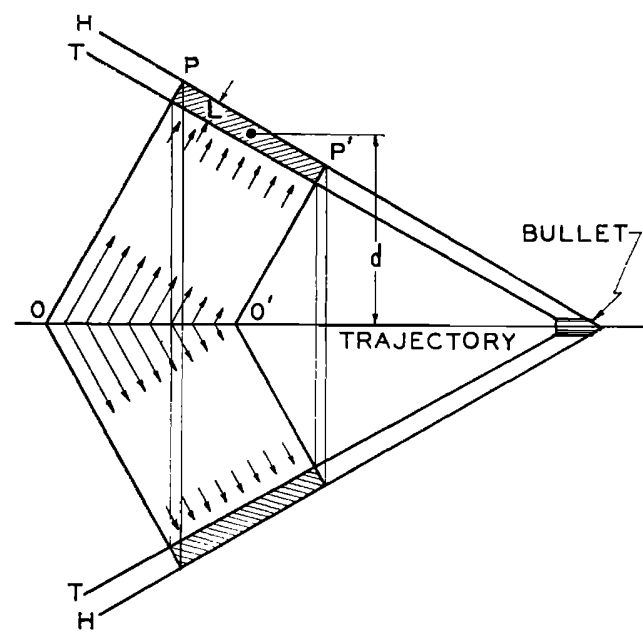

FIG. 5. Path of energy transfer from trajectory to shock wave.

tween $\mathrm{N}$-wave amplitudes, periods, and missdistances come out of the theory and seem also to be consistent with our empirical observations.

Table I gives an iclea of the magnitude of shock wave pressures based on measurements of the rate of change of $\mathrm{N}$-wave period with missdistance. These estimates have been shown to be in accord with condenser microphone measurements if one makes allowance for the reflected wave.

\section{GENERAL DISCUSSION OF THE PROPAGATION AND DISSIPATION OF LOW INTENSITY BALLISTIC SHOCK WAVES}

\section{Plan of Attack}

We shall attack the theory of ballistic $\mathrm{N}$-wave propagation as follows:

1. We first develop very briefly the physical ideas governing the propagation of the so-called "discontinuous" fronts ( $H$ and $T$ of the $\mathrm{N}$-wave profile) and the theoretical formulas for the temperature rise, particle velocity, excess propagation velocity, etc., of these fronts expressed as functions of the pressure jump in them. Only the case of plane fronts will be treated since the thickness of the fronts is very small in comparison to their radii of curvature at the missdistances of interest to us.

2. We next develop by a thermodynamic argument the rate of dissipation of the wave energy in a plane ballistic $\mathrm{N}$-wave (its degrada- tion into heat). This heat is made manifest as a slight temperature elevation of the air at the rear of the $\mathrm{N}$-wave.

3. We then show by an application of Earnshaw's solution for the propagation of finite waves that if the wave form is truly $N$-shaped, with strictly linear intermediate sloping pressure profile, the natural change in slope of this linear portion together with the differential propagation velocities of the two fronts just accounts for the decay in amplitude derived thermodynamically so that if the wave has once assumed the $\mathrm{N}$-form it will thereafter continue to retain it. The argument here is based essentially on plane waves for simplicity.

4. Finally we apply these results to the propagation of $\mathrm{N}$-waves both for the conical ballistic waves from bullet trajectories and also for spherically radiating $\mathrm{N}$-waves such as might occur as the result of explosions. In both of these cases it is assumed that the radii of curvature of the wave surfaces are sufficiently large relative to the wave-length so that the formulas for rate of energy dissipation developed for plane $\mathrm{N}$ waves hold, with sufficient accuracy, for conical and spherical waves. At close miss-distances (the worst case), the wave-length of the $\mathrm{N}$-wave from a $40-\mathrm{mm}$ bullet (measured between $H$ and $T$ discontinuities) is about one foot long or about one-tenth the radius of curvature of the wave surfaces.

Instead of solving the equations of wave propagation in three dimensions, we adopt the easier approximate method of computing the dependence of amplitude on miss-distance by utilizing the principle of conservation of the wave energy (taking into account however the

TABLE I. Absolute ballistic shock wave magnitudes for air.

\begin{tabular}{|c|c|c|c|}
\hline & $\begin{array}{l}\text { Formula for } \\
\text { shock wave } \\
\text { magnitude }\end{array}$ & $\begin{array}{l}40 \mathrm{~mm} \text { at } 40-y d . \\
\text { miss-distance }\end{array}$ & $\begin{array}{l}\text { Ordinary speech } \\
\text { at one yard }\end{array}$ \\
\hline $\begin{array}{l}\text { Peak pressure } \\
\text { elevation }\end{array}$ & $p_{2}-p_{1}$ & $\begin{array}{l}2200 \text { dynes } \mathrm{cm}^{-2} \\
\left(0.032 \mathrm{lb}^{-2} .^{-2}\right)\end{array}$ & $\begin{array}{l}2 \text { dynes } \mathrm{cm}^{-2} \\
\left(0.00003 \mathrm{lb}^{-2} .^{-2}\right)\end{array}$ \\
\hline $\begin{array}{l}\text { Max. particle } \\
\text { velocity }\end{array}$ & $\frac{5}{7} \frac{p_{2}-p_{1}}{p_{1}} c$ & $52 \mathrm{~cm} \mathrm{sec}^{-1}$ & $0.05 \mathrm{~cm} \mathrm{sec}^{-1}$ \\
\hline $\begin{array}{l}\text { Max. tempera- } \\
\text { ture rise }\end{array}$ & $\frac{2}{7} \frac{p_{2}-p_{1}}{p_{1}} T$ & $0.19 \mathrm{C}^{\circ}$ & $0.0002 \mathrm{C}^{\circ}$ \\
\hline $\begin{array}{l}\text { Peak intensity } \\
\text { watts } / \mathrm{cm}^{2}\end{array}$ & $\frac{5}{7} \frac{\left(p_{2}-p_{1}\right)^{2}}{p_{1}} c$ & 0.0115 & $10^{-8}$ \\
\hline $\begin{array}{l}\text { Shock wave } \\
\text { discontinuity } \\
\text { thickness in cm }\end{array}$ & $3.10^{-5}\left(\frac{p_{2}-p_{1}}{p_{1}}\right)^{-1}$ & 0.014 & \\
\hline
\end{tabular}


rate at which it is degraded into heat) as it propagates away from the trajectory between the two cones $O P$ and $O^{\prime} P^{\prime}$ of Fig. 5. The average energy density (in the shaded volume of revolution of that figure) diminishes because of three influences, the first two being merely geometrical increases in the volume containing the energy and the third being the dissipation into heat. The geometrical changes are (1) the increase in the mean radius $d$ and (2) the increase in the wave-length $L$ measured between the discontinuities $H$ and $T$. For the weak shock waves with which we are concerned, the amplitude has been taken as proportional to the square root of the energy density.

\section{Physical Ideas Behind the Theory of Shock Wave Discontinuities}

It is well known that the usual differential wave equation for the propagation of sound waves in air is only an approximation for the case in which the pressure differences in the wave are sufficiently small with respect to existing atmospheric pressure. Here we shall discuss the propagation of waves of finite amplitude in which the first-order correction is retained. The square (and higher powers) of the ratio of wave amplitude to atmospheric pressure is neglected. Specifically, our results shall be applicable to shock waves produced by projectiles at such distances from the source that the pressure elevation in the wave is of the order of 1 percent of an atmosphere or less.

Riemann discovered that the integration of the exact equations led to the possibility, under certain conditions, of the propagation of "a surface of discontinuity" across which such variables as pressure, density, temperature, and particle velocity jump by a finite amount (constituting what is termed mathematically a "first order" discontinuity). ${ }^{2}$ Qualitatively we can understand

2 B. Riemann, "Über die Fortpflanzung ebener Luf twellen von endlicher Schwingungsweite," Göttinger Nachrichten (1860), p. 8; also B. Riemann and H. Weber, Partielle Differential-gleichung, fifth edition, Vol. II., p. 507. In the physical wave the term "discontinuity" is, of course, too strong. As we shall see this "discontinuity" takes the form of an extremely abrupt rise confined to an extremely thin but finite region, whose thickness turns out to be determined by the amplitude of the pressure jump on the one hand and the viscosity and thermal conductivity of the medium on the other hand.

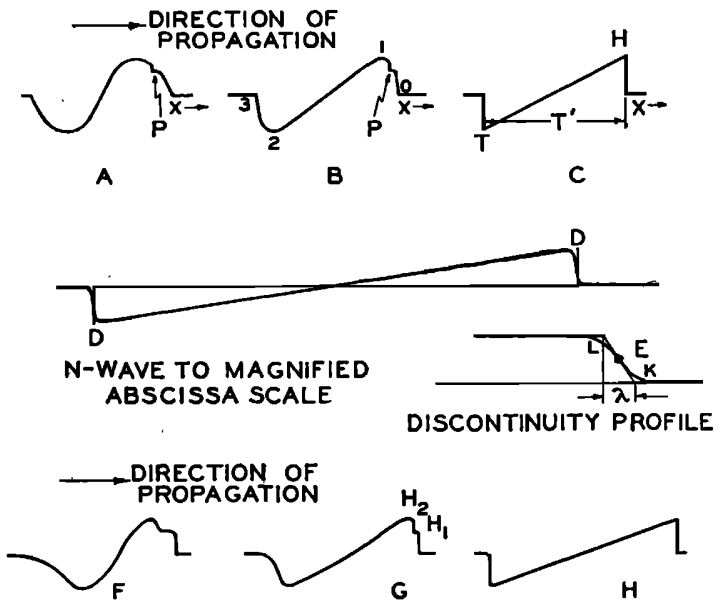

FIG. 6. Evolution of wave forms into N-waves.

the method of production of this discontinuity front by considering a plane one-dimensional acoustic wave which initially has the shape (for example) of a single sinusoidal oscillation.

Figure 6 illustrates such a wave at $A$. We suppose that the curve represents the pressure profile of the wave, the direction of propagation being that shown by the arrow. To clarify the discussion we suppose that this initial wave form has a small nick (at $P$ ) whose history we can follow. The pressure on the $x$ axis is the undisturbed atmospheric pressure.

As the wave propagates to the right the higher amplitude regions propagate more rapidly (relative to the undisturbed medium) than the lower amplitude regions. Regions of the wave form near the $x$ axis propagate nearly at ordinary sonic velocity. Regions of the wave form below the $x$ axis propagate (relative to the undisturbed medium) at less than ordinary sonic velocity. Thus after a lapse of time the wave form will appear as at $B$. The regions $0-1$ and $2-3$ in which the pressure is rising with time become steeper. The region 1-2 in which the pressure is falling with time becomes less steep. ${ }^{3}$

The reason for these differential velocities of propagation is simple. The chief contributing cause is the longitudinal particle velocity or streaming velocity of the air which is always associated with acoustic waves. This streaming

${ }^{3}$ Rayleigh described this process of change of wave form in Theory of Sound (The Macmillan Company, New York, 1896), second edition, Vol. II, pp. 35 and 36. 
velocity is approximately proportional at each point of the wave form to the pressure amplitude at that point. Relative to the undisturbed medium it is directed forward in the positive regions of the wave form and rearward in the negative regions. Thus the small nick in the wave form at $P$ must be regarded as a wavelet which is being propagated to the right in air which itself is moving to the right with a streaming velocity (particle velocity) appropriate to the pressure amplitude at $P$. There is also a higher order effect of less importance coming from the fact that the propagation of the nick occurs in air at higher temperature than the undisturbed medium so that the local propagation velocity there is slightly higher. The mechanisms just described operate in a similar way in the negative regions of the wave form (below undisturbed atmospheric pressure) where the rearward particle velocity and the lowered temperature operate to cause the wave form to lag behind the regions on the $x$ axis.

Now as this mechanism of differential propagation progresses, the rising portions of the waveform must eventually tend to become vertical. An analogous process is familiar in gravity waves on water where the rising regions of the wave profile not only become vertical but pass beyond the vertical so that "breakers" are formed. In the case of the acoustic wave we are discussing, this cannot happen because the wave form is a graph of the pressure and it must therefore be a single valued function of space and time. The acoustic wave profile cannot even become strictly vertical. Some new mechanism must therefore assume importance in the propagation process to place a limitation on the steepness of the nearly discontinuous fronts which the operation of differential propagation speeds has created. At $C$ in Fig. 6 we show the appearance of the wave form after a sufficient amount of propagation has resulted in complete formation of the "discontinuities" $H$ and $T$. In view at $C$ it must be understood that if either discontinuity were examined to a highly magnified scale of abscissae it would be found to have a continuous structure (save for the molecular structure of the gas) as shown in the magnified view $D$. We shall define the "thickness" of the discontinuity by the geometrical construction shown at $E$ as the pro- jection on the $x$ axis of the steepest tangent to the profile.

The new mechanism which becomes the important factor in placing a lower limit on the thickness of the fully formed discontinuity turns out to be the actions of (1) thermal conductivity and (2) viscosity in transferring heat and momentum across the regions of rapid change in temperature and pressure. These two factors tend to blur the sharpness of the front by setting up a flow of heat and momentum from the higher to the lower portions of the wave form. In this way a balance is set up between the steepening action of differential propagation velocity on the one hand and the blurring action of the above two dissipative agencies so that from then on, as long as the amplitude of the discontinuity remains constant, its thickness $\lambda$ remains the same. It turns out that for the weak shock waves we are considering the thickness $\lambda$ is inversely proportional to the amplitude.

For shock waves of amplitude 0.01 atmosphere the thickness $\lambda$ is of order $3 \times 10^{-3} \mathrm{~cm}$. For much more energetic shock waves, R. Becker ${ }^{4}$ computes a "thickness" less than the mean-freepaths of air molecules. ${ }^{5}$

It should be noted that the $\mathrm{N}$-shaped wave profile we have described would be the end result of a large variety of other initial wave forms and that the assumption of the sinusoidal shape shown at $A$ was not necessary. At $F, G$, and $I I$, we show the evolution of another wave form to the same end result. It is perfectly possible to have two discontinuities, $H_{1}$ and $H_{2}$ as at $G$, which eventually merge into one because of the faster propagation of $H_{2}$ than $H_{1}$. The merging of two such steps can be clearly seen on the direct photographs of ballistic shock waves.

Once a discontinuity has reached its final

\footnotetext{
${ }^{4} \mathrm{R}$. Becker "Stosswelle und Detonationen," Zeits. f. Physik 8, 321-347 (1921).

5 In Lamb's Hydrodynamics (Dover Publications, New York, 1945), sixth revised edition, Sections $360 \mathrm{a}$ and $281 \mathrm{et}$ seg., respectively, formulas are developed for the profile structure of shock wave discontinuities, for two hypothetical cases (1) viscosity but no thermal conduction and (2) thermal conduction but no viscosity. For weak shock waves the profile assumes the shape of a curve of hyperbolic tangents. L. H. Thomas [J. Chem. Phys. 12, 449 (1944)] shows how the computation of the wave front "thickness" may be extended to the cases of the most intense explosions, by taking into account the variation of heat conductivity and of viscosity with temperature.
} 
equilibrium state (through the balance of steepening and blurring tendencies above described) it is propagated from then on at a characteristic velocity definitely related to its amplitude. The fact that once this balance is attained the profile of the discontinuity is thenceforth stable in time makes derivation of the formulas relative to such discontinuities very simple. This is done by assuming coordinates in which the profile is stationary and writing the equations of continuity of mass, energy, and momentum relative to a sample of gas as it moves through the discontinuity.

As a sample of the gas rises up the pressure hill in its passage through the discontinuity the transfer of heat through thermal conduction results in an important change of entropy. In Fig. 6, at $E$ the gas entering at $K$ receives heat by conduction from the closely adjacent hotter gas at $L$. The heated sample then undergoes compression and as soon as it has reached the region $L$ it gives up heat at its more elevated temperature to the new gas entering at $K$. This process results in a higher final temperature for the same pressure jump than would be the case without heat transfer. It has been called a "dynamic adiabatic" process ${ }^{6}$ to distinguish it from the more familiar static adiabatic process in which pressure and temperature ratios are related by the classical law

$$
p T^{\gamma /(1-\gamma)}=\text { a constant. }
$$

\section{MATHEMATICAL THEORY}

\section{Discontinuous Changes in Properties Across a Shock Wave Front ${ }^{7}$}

We shall now consider a simple pressure pulse (consisting of a change from initial pressure $p_{0}$ to a pressure $\left.p_{1}\right)$ whose amplitude $\left(p_{1}-p_{0}\right)$ is maintained at some constant value. No matter what was the original form of the pressure profile eventually a single discontinuous step will be

\footnotetext{
- The dynamic adiabatic compression here described was apparently first discussed by Hugoniot, J. de l'Ecole Polytech. Paris 57 (1887) and 58 (1889). The propriety of the word adiabatic may be questioned since heat transfer takes place. However no heat is communicated to the gas from external sources.

${ }^{7}$ The development of this section follows R. Becker, "Stosswelle und Detonationen," Zeits. f. Physik 8, 321 (1921) and is reproduced briefly here for clarity and completeness.
}

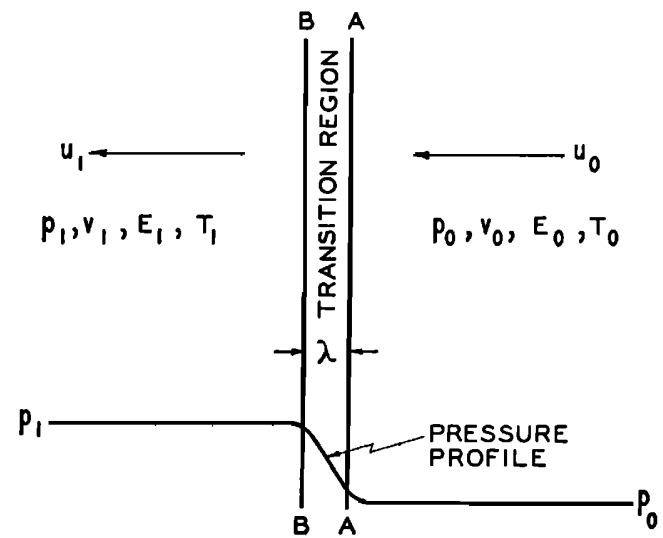

FIG. 7. Passage of gas through a Riemann "discontinuity" (nomenclature).

formed as we have explained. Such a step is shown in Fig. 7. Since the propagation velocity of the discontinuity is essentially constant and the profile does not change with time we can choose coordinates in which the wave front is stationary. The gas then streams in from the right with a velocity $u_{0}$, numerically equal to the velocity of propagation of the wave in still air. As the air passes through the discontinuity it loses kinetic energy so that on the left its streaming velocity is $u_{1}\left(<u_{0}\right)$. Viewed in coordinates in which the wave is moving (with velocity $u_{0}$ ) and the air is initially at rest, this means that behind the wave the air possesses a particle velocity $W=u_{0}-u_{1}$ in the direction of propagation of the wave. In Fig. 7, the region between the planes $A, B$ (seen on edge) is the region of discontinuity. The pressure distribution is indicated at the bottom, and a similar curve can be drawn for each of the other variables such as temperature, density, etc. We shall designate all quantities on the right by the subscript 0 and all quantities on the left by the subscript 1 . The pressure is designated by $p$, the specific volume by $v$, the absolute temperature by $T$, the air velocity by $u$, and the intrinsic energy density (per unit mass) as $E$. The laws of conservation of mass, momentum and energy require the fulfillment respectively of the equations

$$
\begin{aligned}
u_{1} / v_{1} & =u_{0} / v_{0}, \\
u_{1}^{2} / v_{1}+p_{1} & =u_{0}^{2} / v_{0}+p_{0}, \\
E_{1}+\frac{1}{2} u_{1}^{2}+p_{1} v_{1} & =E_{0}+\frac{1}{2} u_{0}^{2}+p_{0} v_{0} .
\end{aligned}
$$


Furthermore we have the equation of state of the gas, which we shall consider to be ideal,

$$
\begin{gathered}
p v=R T, \\
p_{1} v_{1} / T_{1}=p_{0} v_{0} / T_{0}=R, \\
E_{0}-E_{1}=c_{v}\left(T_{0}-T_{1}\right) .
\end{gathered}
$$

Clearly our problem involves the ten quantities shown in Fig. $7, u_{0}, p_{0}, v_{0}, E_{0}, T_{0}, u_{1}, p_{1}, v_{1}, E_{1}$, $T_{1}$, five of which $p_{1}, p_{0}, v_{0}, E_{0}, T_{0}$ are to be regarded as given. The five equations (1)-(5) are therefore sufficient for a complete determination of the remaining five quantities $u_{0}, u_{1}, v_{1}$, $E_{1}$, and $T_{1}$.

It is important to realize clearly that no additional assumption has been made, nor can be made, regarding the thermodynamic "path" of the gas as it undergoes the compression (such for example, as the assumption that the gas, besides obeying Eqs. (4) and (5), is compressed according to the familiar law $p v^{\gamma}=\mathrm{a}$ constant, the so-called adiabatic compression taking place by a slow quasi-static process). Any such assumption would be redundant since the five fundamental conditions above are necessary and sufficient in themselves to determine the final state of the gas and they do in fact determine a final state which for intense shock waves is not that which the gas would have reached by the path $p v^{\gamma}=\mathrm{a}$ constant. (For the ballistic shock waves with which we are now concerned however, where $\left(p_{1}-p_{0}\right) /$ $p_{0}$ never much exceeds 0.01 atmosphere, the deviation from the static adiabatic law, $p v^{\gamma}=$ constant, is extremely small indeed and for some purposes completely negligible.)

We solve Eqs .(1) to (5) adopting the abbreviated nomenclature

$$
\zeta=2 c_{v} / R+1=(\gamma+1) /(\gamma-1),
$$

wherein $\gamma$ is the specific heat ratio. For air, since $\gamma=1.4, \zeta=6$.

If

$\pi=p_{1} / p_{0}$ (the shock-wave pressure in atmospheres)

we obtain the following exact equations:

The temperature ratio

$$
\frac{T_{1}}{T_{0}}=\pi-\frac{\pi+\zeta}{\pi \zeta+1}
$$

The specific volume or density ratio

$$
\frac{v_{0}}{v_{1}}=\frac{\rho_{1}}{\rho_{0}}=\frac{\pi \zeta+1}{\zeta+\pi} .
$$

The discontinuity propagation velocity relative to still air, $D=\left|u_{0}\right|$, is given by the equation:

$$
D^{2}=\rho_{0} v_{0} \frac{\pi \zeta+1}{\zeta-1} .
$$

The particle velocity $W$ behind the moving front $\left(=u_{1}-u_{0}\right)$ is given by

$$
W^{2}=p_{0} v_{0}(\zeta-1) \frac{(\pi-1)^{2}}{\pi \zeta+1}
$$

Since we are only to be concerned with relatively weak shock waves in which the variable $\pi=p_{1} / p_{0}$ differs from unity by never much more than 1 percent, the above formulas can be greatly simplified by substituting for $\pi$ the value $1+\delta$, expanding the expressions in powers of $\delta$, and rejecting terms of the second and higher orders. Clearly $\delta=\left(p_{1}-p_{0}\right) / p_{0}$. We thus finally obtain the following very simple and useful expressions for weak shock waves relating other shock wave discontinuity magnitudes to the relative pressure rise in a shock wave discontinuity. They are conveniently expressed in terms of relative changes. The relative temperature rise is

$$
\frac{T_{1}-T_{0}}{T_{0}}=\frac{\gamma-1}{\gamma} \frac{p_{1}-p_{0}}{p_{0}} .
$$

The relative change in specific volume or density

$$
\frac{v_{0}-v_{1}}{v_{1}}=\frac{\rho_{1}-\rho_{0}}{\rho_{0}}=\frac{1}{\gamma} \frac{p_{1}-p_{0}}{p_{0}} .
$$

The relative excess propagation velocity of the discontinuity

$$
\frac{D-c}{c}=\frac{\gamma+1}{4 \gamma} \frac{p_{1}-p_{0}}{p_{0}}
$$

( $c=$ ordinary velocity of infinitesimal sounds).

The particle velocity $W$ immediately behind the front

$$
W=\frac{1}{\gamma} \frac{p_{1}-p_{0}}{p_{0}}
$$


The intensity

$$
I=\frac{1\left(p_{1}-p_{0}\right)^{2}}{\gamma}-c \frac{p_{0}}{\gamma} .
$$

\section{A Thermodynamic Method of Computing the Energy Dissipation (with Propagation) of Plane Ballistic Shock Waves in Air}

It is empirically observed that the wave forms of the shock waves generated by bullets resemble the curve of Fig. 8 (herein referred to as the "N-wave"). The vertical segments of the curve represent extremely abrupt rises of pressure, particle velocity, temperature, or condensation. The region in which this nearly discontinuous rise occurs is extremely short compared to the distance between the two discontinuities 0,1 and 3 , 4. The pressure amplitudes of the two discontinuities are nearly equal and of the order of 0.02 atmosphere at four meters from the trajectory in the case of a $40-\mathrm{mm}$ bullet. The part of the wave form 1, 2, 3 above is a monotonically descending curve which can be well approximated by a straight line. Let us designate the values of all variables such as pressure $p$, temperature $T$, etc., at each point of the curve by a subscript number referring to the number of the point in Fig. 8. Then $p_{0}$ is the undisturbed atmospheric pressure.

Assumption I: The pressure $p_{4}$ at 4 equals $p_{0}$ and the wave form is logarithmically balanced ${ }^{8}$ $\left(p_{1} / p_{0}=p_{4} / p_{3}\right)$.

Assumption II: We assume that the above wave form has propagated sufficiently far so that the discontinuities have reached their quasiequilibrium state of maximum steepness. (This is the state in which the steepening tendency (caused by the excess speed of propagation of the higher amplitude portions of the wave form) is just balanced by the blurring tendency of the gas-kinetic thermal and momentum transport across the thin front, as already explained in the previous section.)

When this quasi-equilibrium state has been reached, the wave form in the transition regions of the discontinuities is almost stable and we shall make:

\footnotetext{
${ }^{8}$ For weak shock waves in which $\left(p_{1}-p_{0}\right) / p_{0} \ll 1$ this is substantially the same as saying that $\left(p_{1}-p_{0}\right)=\left(p_{4}-p_{3}\right)$ so that logarithmic and arithmetic balance are practically equivalent conditions.
}

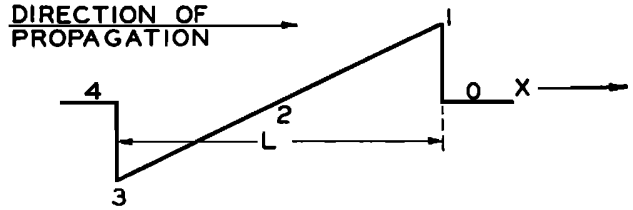

FIG. 8. N-wave nomenclature for derivation of thermal dissipation formulas.

Assumption III : That the wave form of the discontinuity can be treated for the purpose of computing the dissipation as constant in time.

Assumption IV: The entropy of a sample of the gas in passing from the state 1 to the state 2 and thence to the state 3 does not change. (It is known that the entropy increases in going from state 0 to state 1 and also in going from state 3 to state 4.)

According to the received theory for the propagation of such quasi-stable discontinuities as 0 , 1 or 3,4 , a sample of the gas passing through such a discontinuity undergoes change of state subject to the laws of conservation of mass, momentum, and energy and since the wave form is not changing sensibly with time there result, as shown in the previous section, certain simple formulas for the condensation ratio $\left(\rho_{1}-\rho_{0}\right) / \rho_{0}$, the temperature ratio $T_{1} / T_{0}$, the particle velocity, etc., all such variables being expressible in terms of one parameter which we shall take as the pressure ratio $p_{1} / p_{0}$ (or $\left.p_{4} / p_{3}\right)$.

The wave energy dissipated with propagation of the wave form must of necessity manifest itself in the immediate wake of the wave at 4 . It can appear there in only three forms, thermal, kinetic, or potential, i.e., the gas at 4 may be hotter than at 0 , it may be possessed of a body velocity (relative to the gas at 0 ), or the pressure at 4 may exceed the pressure at 0 . The last of these three possibilities is ruled out by our Assumption I, and we shall later show that, to $a$ first approximation, there is no body velocity at 4 ascribable to energy lost by the $\mathrm{N}$-wave.

We shall now compute the thermal part of the dissipated energy.

Let the pressure ratio $p_{i} / p_{j}$ for any two points $i$ and $j$ on the wave form be expressed as $\pi_{i j}$. Now according to the received theory (Section I) for the propagation of acoustic discontinuities we have for the ratio of the initial and final 
temperatures the following exact expression (Eq. (8), Section I).

$$
\frac{T_{1}}{T_{0}}=\pi_{10} \frac{\pi_{10}+\zeta}{\pi_{10} \zeta+1},
$$

(where $\zeta=2 c_{v} / R+1=(\gamma+1) /(\gamma-1)=6$ for a diatomic gas).

Similarly for the second discontinuity we have

$$
\frac{T_{4}}{T_{3}}=\pi_{43} \frac{\pi_{43}+1}{\pi_{43} \zeta+1} .
$$

The gas, on the other hand expands isentropically and reversibly in passing from state 1 to state 3 . It follows the law

$$
T_{3} / T_{1}=\pi_{31}{ }^{2 /(\zeta+1)} .
$$

Now by taking the product of expressions (1)(3) we obtain

$\frac{T_{4}}{T_{0}}=\frac{T_{1}}{T_{0}} \frac{T_{3}}{T_{1}} \frac{T_{4}}{T_{3}}=\pi_{10} \pi_{31} \pi_{43} \frac{\pi_{10}+\zeta}{\pi_{10} \zeta+1} \frac{\pi_{43}+\zeta}{\pi_{43} \zeta+1} \pi_{31}{ }^{(1-\zeta) /(1+\zeta)}$.

But since $\pi_{43}=\pi_{03}$ (Assumption I) it follows that $\pi_{10} \pi_{31} \pi_{43}=1$ and also $\pi_{31} \equiv \pi_{30} \pi_{01} \equiv \pi_{03}{ }^{-1} \pi_{10}{ }^{-1}$ so that we can write, in general,

$\frac{T_{4}}{T_{0}}=\frac{\pi_{10}+\zeta}{\pi_{10} \zeta+1} \pi_{10}(\zeta-1) /(\zeta+1) \cdot \frac{\pi_{03}+\zeta}{\pi_{03} \zeta+1} \pi_{03}(\zeta-1) /(\zeta+1)$

and in particular if the $\mathrm{N}$-wave is "balanced" logarithmically, that is, if $\pi_{10}=\pi_{03}=\pi$ then

$$
\frac{T_{4}}{T_{0}}=\left[\frac{\pi+\zeta}{\pi \zeta+1}\right]^{2} \pi^{2(\zeta-1) /(\zeta+1)}
$$

The above expression is exact. It becomes of interest however to simplify this expression by an approximation for the case in which $\pi$ differs little from unity as is the case in ballistic shock waves.

$$
\text { Let } \pi_{i j}=1+\delta_{i j} ; \delta \ll 1 \text {. }
$$

If we make this substitution in (4.1), we obtain after expanding in powers of $\delta$

$$
\begin{aligned}
& \frac{T_{4}}{T_{0}}=\left[1+\frac{\zeta(\zeta-1)}{3(1+\zeta)^{3}} \delta^{3}+\cdots\right]^{2} \\
& =1+\frac{2}{3} \frac{\zeta(\zeta-1)}{(1+\zeta)^{3}} \delta^{3}+\cdots=1+\Delta .
\end{aligned}
$$

We have then for the temperature rise at 4 the following expression

$$
T_{4}-T_{0}=\Delta \cdot T_{0} ; \quad \Delta=\frac{2}{3} \frac{\zeta(\zeta-1)}{(1+\zeta)^{3}} \cdot \delta^{3} .
$$

The air in its initial state is at temperature $T_{0}$ and pressure $p_{0}$. After the wave has swept over and communicated heat to the air, the latter is left at a higher temperature $T_{4}$ but the same pressure $\mathrm{p}_{0}$. The thermal power loss $d W / d t$ (per unit cross section of wave front) associated with this heating at constant pressure is therefore

$$
\text { Power loss }=\Delta \cdot T_{0} \cdot c_{p} \cdot p_{0} \cdot c=d W / d t \text {. }
$$

The total energy (potential plus kinetic) associated with the $\mathrm{N}$-wave (per unit cross section of wave front) is

$$
W=\text { wave energy }=p_{0} \delta^{2} L / 3 \gamma,
$$

wherein $L$ is the wave-length measured between the discontinuities. The fraction of the wave energy lost per unit distance the wave is propagated is therefore

$$
\frac{d W}{d x} \frac{1}{W}=\frac{1}{c} \frac{d W}{d t} \frac{1}{W}=2 \frac{\zeta(\zeta-1)}{(1+\zeta)^{3}} \delta \gamma \frac{T_{0} c_{p} \rho_{0}}{p_{0} L} .
$$

Since

$$
\frac{T_{0} \rho_{0}}{p_{0}}=\frac{1}{R}=\frac{\zeta+1}{2 c_{p}},
$$

we can write (9) as

$$
\frac{d W}{d x} \frac{1}{W}=\frac{\zeta}{\zeta+1} \frac{\delta}{L}=\frac{\gamma+1}{2 \gamma} \frac{\delta}{L}=\frac{6}{7} \frac{\delta}{L} \text { for air. }
$$

It is interesting to note that the derivation of this formula involves no assumptions regarding (1) the wave form in the abrupt transition regions and (2) the coefficients of viscosity and thermal conductivity. All such considerations are cancelled out. The thermal losses are occasioned by the irreversible changes of state occurring in the abrupt transitions 0,1 and 3,4 and these changes are completely determined once the pressure amplitudes are given. An interesting conclusion from these considerations is that the thermal loss in an $\mathrm{N}$-shaped ballistic shock wave depends only on $\gamma$, the specific heat ratio of the gas and is otherwise independent of the nature 
of the gas. Among gases having the same $\gamma$, those having higher thermal conductivity have broader transitions, 0,1 and 3,4 so as to maintain the same thermal loss.

Furthermore the above expression represents the full amount of energy dissipation for we shall now show that there is no particle velocity behind the $\mathrm{N}$-wave relative to the undisturbed medium in front, and therefore there is no transfer of energy from the $\mathrm{N}$-wave into kinetic energy of the gas behind the wave.

From the constitution of the gas there is nothing to prevent assigning arbitrary values to both the density $\rho$ and particle velocity $u$, but for a progressive wave a relation between these two must exist. Earnshaw has shown ${ }^{9}$ that this relation for the case of a wave of finite amplitude is

$$
u=\int\left(\frac{d p}{d \rho}\right)^{\frac{1}{2}} \frac{d \rho}{\rho}
$$

Therefore, we may, in a manner similar to that used above for the temperature, determine the net change in particle velocity brought about by the passage of the $\mathrm{N}$-wave.

Using a notation similar to that used in the first part of this section, we have (from Eq. (11) or (11.1), Section I) for the change in particle velocity across the discontinuities

$$
u_{1}-u_{0}=\frac{c_{0}}{\gamma} \frac{p_{1}-p_{0}}{p_{0}} \text { and } u_{4}-u_{3}=\frac{c_{3}}{\gamma} \frac{p_{4}-p_{3}}{p_{3}}
$$

where $c_{0}$ and $c_{3}$ are the local propagation velocities of infinitesimal waves under the conditions existing at points 0 and 3 .

To compute the change in velocity over the adiabatic portion of the path $1,2,3$, we must use Earnshaw's equation presented above. Over this region we have the adiabatic relationship between $\rho$ and $p$ namely

$$
\rho=\rho_{1}\left(\frac{p}{p_{1}}\right)^{1 / \gamma}, \frac{d \rho}{d p}=\frac{\rho_{1}}{\gamma p_{1}}\left(\frac{p}{p_{1}}\right)^{(1-\gamma) / \gamma},
$$

so that using Earnshaw's relation

$$
u=\left(\frac{\gamma p_{1}}{\rho_{1}}\right)^{\frac{1}{2}} \int\left(\frac{p}{p_{1}}\right)^{(\gamma-1) / 2} \frac{d p}{\gamma p}
$$

\footnotetext{
${ }^{9}$ Earnshaw, Phil. Trans., 146 (1859).
}

and

$$
u_{3}-u_{1}=\left(\frac{\gamma p_{1}}{\rho_{1}}\right)^{\frac{1}{\gamma}} \frac{2}{\gamma-1}\left[\left(\frac{p_{3}}{p_{1}}\right)^{(\gamma-1) / 2 \gamma}-1\right] .
$$

If we expand the above expressions and keep only the first powers in $\delta=\left(p_{1}-p_{0}\right) / p_{0}$, we find

$$
\begin{aligned}
& u_{1}-u_{0}=c_{0} \delta / \gamma, \\
& u_{3}-u_{1}=-2 c_{0} \delta / \gamma, \\
& u_{4}-u_{3}=c_{0} \delta / \gamma
\end{aligned}
$$

adding these three equations we find

$$
u_{4}-u_{0}=0 \text {, }
$$

so that the particles behind the $\mathrm{N}$-wave have the same velocity as particles in front of the $\mathrm{N}$-wave and therefore, as stated above, there is no transfer of energy from the $\mathrm{N}$-wave into kinetic form. Furthermore, it is clear that if we had retained terms of order $\delta^{2}$, the velocity remaining after passage of the $\mathrm{N}$-wave could not have been represented by a term of lower order than $\delta^{2}$ and hence the dissipation in the form of kinetic energy could not have been of lower order than $\delta^{4}$. The thermal dissipation (see Eq. (5) of this section) is however of order $\delta^{3}$ so that the dissipation in the form of kinetic energy is negligible in comparison thereto.

\section{An Alternate Hydrodynamic Method of Computing the Amplitude Decay of Plane Ballistic Shock Waves in Air}

We shall now present an alternate method for computing the rate of amplitude attenuation of an N-shaped plane wave. As in the previous section we shall use Eqs. (8.1), (9.1), (10.1), (11.1) derived from the theory developed by Becker ${ }^{7}$ for the discontinuities of the $\mathrm{N}$-wave, and the usual adiabatic laws for finite waves for the intermediate portion of the wave. We shall make the same basic assumptions here as were made in Section II, but it should be noted that Assumption III applies only to the wave form of the discontinuities. These latter, as already explained, preserve their wave forms and propagate at their own peculiar velocities as given in Section I, Formula (10) or (10.1). We must, however, allow the linear portion of the wave $(1,2,3$ in Fig. 8) to change its form (slope) with time 
and will correlate this change with the propagation of the discontinuities.

In order to cletermine the behavior of the $\mathrm{N}$ wave, we must therefore consider the hydrodynamics of the adiabatic or linear portion (1, 2,3 , Fig. 8 ) of the $\mathrm{N}$-wave as well as that of the discontinuities: the latter has already been accomplished in Section I; the former will now be developed.

Consider a section of gas of thickness $\xi$ which is constructed in such a way as to include a constant mass of gas. Let the pressure, density, and particle velocity of the gas at one surface be $p, \rho$, and $u$; and let the same variables at the other surface be $p+\xi \partial p / \partial x, \rho+\xi \partial \rho / \partial x$, and $u+\xi \partial u / \partial x$. Furthermore $d \xi / d t=\xi \partial u / \partial x$.

We have three conservation laws which must be met; namely, the conservation of mass, momentum, and energy. The mass of gas included between the planes is $\rho \xi$ and its momentum is $\rho \xi u$ so that the first two laws become

$$
\begin{aligned}
& \frac{d}{d t}(\rho \xi)=0, \\
& \frac{d}{d t}(\rho \xi u)=-\frac{\partial p}{\partial x} \xi .
\end{aligned}
$$

For the third law we shall use the adiabatic gas law which essentially specifies the relation which must exist between the pressure and density of a perfect gas in order that no energy is lost or gained by the gas. In the linear portion the gas expands slowly and with negligible heat transfer between adjacent portions so that

$$
\rho=\rho_{0}\left(p / p_{0}\right)^{1 / \gamma}
$$

in which $\rho_{11}$ and $p_{0}$ refer to the density and pressure at the center of the $\mathrm{N}$-wave where the pressure is equal to that of the undisturbed air. The density at this point differs from the density of the undisturbed air only by terms of the third order in $\delta$, and therefore the difference may be neglected in our approximation.

We obtain, on expanding Eq. (1)

or

$$
\underset{\xi \frac{\partial \rho}{\partial t}}{\partial t} \underset{\partial \rho}{\partial \rho}+\rho \frac{d \xi}{d t}=0
$$

so that

$$
\underset{\partial t}{\frac{\partial \rho}{\xi}+u \xi_{\partial x}^{\partial \rho}}+\rho \xi_{\partial x}^{\partial u}=0
$$

$$
\frac{\partial \rho}{\partial t}+u \frac{\partial \rho}{\partial x}+\rho \frac{\partial u}{\partial x}=0 .
$$

While Eq. (2) becomes, when combined with (1),

$$
\frac{\partial u}{\partial t}+\rho u \frac{\partial u}{\partial x}=-\frac{\partial p}{\partial x} .
$$

If we now multiply Eq. (1.1) by $u$ and combine with (2.1) we obtain

$$
u^{2} \frac{\partial \rho}{\partial x}-\frac{\partial p}{\partial x}=\rho \frac{\partial u}{\partial t}-u \frac{\partial \rho}{\partial t},
$$

which we may write as

$$
\left[u^{2} \frac{d \rho}{d p}-1\right] \frac{\partial p}{\partial x}=\rho \frac{\partial u}{\partial t}-u \frac{d \rho}{d p} \frac{\partial p}{\partial t} .
$$

By the use of (3) Earnshaw's relationship given above takes the form

$$
u-u_{0}=\frac{2 c}{\gamma-1}\left(\frac{p}{p_{0}}\right)^{(\gamma-1) / 2 \gamma}
$$

in which $c=\left[(d p / d \rho)_{0}\right]^{\frac{1}{2}}=\left[\gamma p_{0} / \rho_{0}\right]^{\frac{1}{2}}$, the velocity of propagation of infinitesimal waves in standard air. Putting this expression into Eq. (4.1) we get an expression connecting the rate at which the pressure changes from point to point along the wave at a fixed instant of time and the rate at which the pressure changes with time at a fixed point.

$$
\begin{aligned}
& \left\{\left[\frac{2}{\gamma-1}\left(\frac{p}{p_{0}}\right)^{(\gamma-1) / 2 \gamma}+\frac{u_{0}}{c}\right]^{2}\left(\frac{p}{p_{0}}\right)^{(1-\gamma) / \gamma}-1\right\} \frac{\partial p}{\partial x} \\
& =\frac{1}{c}\left\{\left(\frac{p}{p_{0}}\right)^{(1-\gamma) / 2 \gamma}-\left[\frac{2}{(\gamma-1)}\left(\frac{p}{p_{0}}\right)^{(\gamma-1) / 2 \gamma}+\frac{u_{0}}{c}\right]\left(\frac{p}{p_{0}}\right)^{(1-\gamma) / \gamma}\right\} \frac{\partial p}{\partial t} .
\end{aligned}
$$




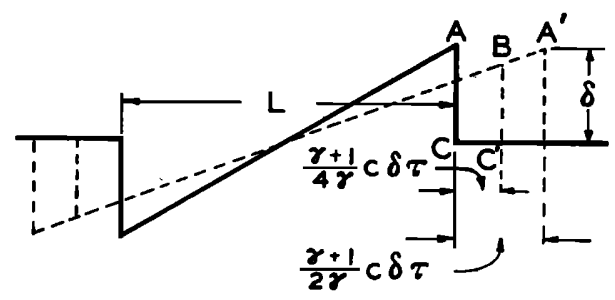

FIG. 9. Diminution of $\mathrm{N}$-wave amplitude by hydrodynamic considerations.

Now we wish to consider the $\mathrm{N}$-wave in coordinates with respect to which the wave form is approximately stationary. At some central point the $\mathrm{N}$-wave has a velocity relative to still air equal to $c$ and the particle velocity at this point is zero, for it seems safe to assume that the propagation velocity of a point on the wave will be $\left[(d p / d \rho)_{0}\right]^{\frac{1}{2}}$ if the particle velocity vanishes at that point. To produce a stationary wave we must therefore impose a velocity $-c$ on the entire wave and we have the condition

Therefore

$$
u=-c \text {, when } p=p_{0} \text {. }
$$

$$
-c-u_{0}=\frac{2 c}{\gamma-1} \text { or } \quad u_{0}=-\frac{\gamma+1}{\gamma-1} c .
$$

If, furthermore, we put $p=p_{0}(1+\delta)$, Eq. (4.2) becomes, upon expansion and dropping terms in $\delta^{2}$,

$$
-\frac{\gamma+1}{2 \gamma} c \delta \frac{\partial p}{\partial x}=\frac{\partial p}{\partial t}
$$

Now

$$
-\frac{\frac{\partial p}{\partial t}}{\frac{\partial p}{\partial x}}=\left.\frac{\partial x}{\partial t}\right|_{p=\text { const. }}
$$

which is the velocity at which a point of constant pressure in the linear intermediate part of the $N$-wave is propagated away from the center of the $N$-wave, or

$$
u_{p}=\frac{\gamma+1}{2} \delta c
$$

The meaning of this is to be interpreted as follows : $u_{p}$ is the propagation velocity (relative to our coordinate system in which the entire wave form is nearly stationary) of any point in the pressure profile of the wave. If we plot this pressure profile for a given instant of time $t$, points on the profile where the pressure amplitude is zero $(\delta=0)$ will have zero velocity, points of positive amplitude will progress to the right, the faster the higher their amplitude, while points of negative amplitude will retrogress toward the left so that at a later instant $t_{2}$ the entire profile, if initially linear, will have undergone an expansion at the rate $(\gamma+1) \delta c / \gamma$ by which we mean that any two points in the profile, of amplitudes, respectively, $\delta$ and $-\delta$ and hence symmetrically situated at equal distances in front and behind the center point $(\delta=0)$, will spread apart at the rate just stated. The slope of the linear portion of the $\mathrm{N}$-wave will thus become less and less steep as the wave progresses.

This velocity $u_{p}$ (Eq. (6)) is just twice the velocity of propagation of the discontinuity as developed previously (Eq. (10.1), Section I). That is, the head and tail discontinuities of the $\mathrm{N}$ wave separate from each other at just half the rate at which points adjacent to them on the linear portion of the wave form separate. This effect must produce a decrease in the amplitude of the $\mathrm{N}$-wave. To see this, consider two successive $\mathrm{N}$-wave shapes (Fig. 9). In a short interval of time, $\tau$, the linear slope of the $\mathrm{N}$-wave spreads out from the solid line to the dotted line. Were it not for the fact that the discontinuity is propagated at a velocity which is less than the propagation velocity of the point $A$ on the linear portion, this point would move to the position $A^{\prime}$. The discontinuity, $A C$, has moved only to the position $B C^{\prime}$, so that the point $A$ is forced to move to the position $B$ and the amplitude of the Nwave has consequently decreased. This decrease can easily be calculated from the geometry of the above figure. We obtain immediately

$$
\frac{d \delta}{d t}=-\frac{(\gamma+1) c \delta^{2}}{2 L},
$$

since $\tau=d t$. The energy in the $\mathrm{N}$-wave is proportional to the wave-length $L$, and to the square of the amplitude, $\delta$, so that differentiating logarithmically we find that the fractional change 
in energy is

$$
\frac{d W}{d t} \frac{1}{W}=\frac{d L}{d t} \frac{1}{L}+2 \frac{d \delta}{d t} \frac{1}{\delta}
$$

The wave-length is changing owing to the propagation of the head discontinuity at a velocity greater than that of infinitesimal sounds and of the tail wave at a velocity less than the velocity of sound (relative to the initially undisturbed air). The net result is that

so that

$$
\frac{d L}{d t}=\frac{(\gamma+1)}{2 \gamma}-c \delta,
$$

or

$$
\frac{d W}{d t} \frac{1}{W}=-\frac{(\gamma+1) c}{2 \gamma L} \delta
$$

$$
\frac{d W}{d x} \frac{1}{W}=-\frac{(\gamma+1) \delta}{2 \gamma L},
$$

which represents the rate of loss of energy per unit distance of propagation normal to the wave front and is in agreement with the result obtained in Section II (Eq. (10)).

The calculation of the previous section (II) is based entirely on thermal considerations and makes no assumptions as to the shape of the $N$ wave (except that discontinuities exist, connected by a region in which the changes in air pressure, density, etc., are adiabatic). The thermal argument does not require that the intermediate adiabatic part of the profile be linear. On the other hand the assumptions made in this section are entirely hydrodynamical and the dissipation is developed from purely geometrical arguments based on the linearity of the intermediate part of the wave form. The agreement between the two results indicates that the assumed geometry of the $\mathrm{N}$-wave (particularly the linear fall in pressure between the head and tail discontinuities) enjoys some peculiar privilege as to permanence of form. In fact, if the pressure variation between discontinuities were not linear (for example, if it were sinusoidal) the decrease in amplitude would not be such as to give the dissipation calculated from thermal considerations and there would therefore occur a progressive deformation of wave form until the linear shape was attained.
We may summarize our results then by stating that the $\mathrm{N}$-wave, as it propagates, undergoes an expansion of the total wave-length $L$ at the rate

$$
\frac{d L}{d t}=\frac{(\gamma+1)}{2 \gamma} \delta c,
$$

while the intermediate linear portion in the region between the discontinuities expands at double this rate.

\section{Dependence of Amplitude and Wave-Length of Two-Dimensional Ballistic Shock Waves on Miss-Distance}

We are now in a position to calculate the amplitude and wave-length of a ballistic shock wave as a function of the distance it has propagated. For convenience the results will be expressed in terms of, $y$, the miss-distance measured normal to the trajectory of the bullet.

The diagram, Fig. 10, indicates a segment $O O^{\prime}$ of the trajectory of a bullet which we shall take of unit length. The energy given to the shock wave by the bullet as it traversed this segment $O O^{\prime}$ will remain confined between two coaxial cones formed by rotating $O P$ and $O^{\prime} P^{\prime}$ about the trajectory as an axis. At the moment indicated in the diagram, the energy of the shock wave is entirely within the ring of radius $y$ whose section is the shaded area in the figure.

The total energy in this ring is the volume of the ring multiplied by the average energy density. It can easily be shown that for a linear, "N," wave form such as we have postulated, the average energy density is

so that

$$
W_{a r}=p_{0} \delta^{2} / 3 \gamma,
$$

$$
W=2 \pi y \cos \theta L\left(p_{0} \delta^{2} / 3 \gamma\right) .
$$

As the $\mathrm{N}$-wave is propagated away from the trajectory in the direction $x$ as shown in Fig. 10

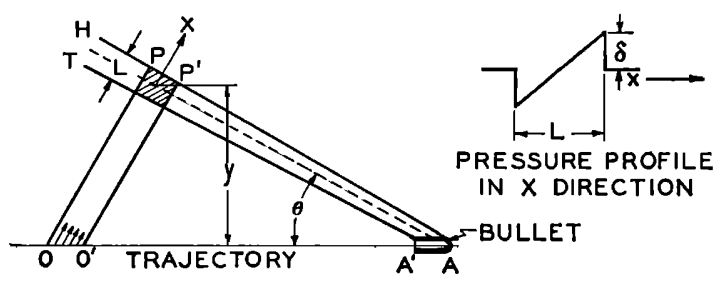

FIG. 10. Geometry for derivation of amplitude dependence on miss-distance 
(i.e., normal to the wave front) three factors contribute to the decrease in the energy density in the wave and hence to the decrease in amplitude $\delta$. Two of these factors are purely geometrical. (1) The ring, above referred to, whose cross section is the shaded rectangle in Fig. 10, has a continually increasing radius $y$ and hence a proportionally increasing volume over which the wave energy $W$ is distributed. (2) The wavelength $L$ of the $\mathrm{N}$-wave is subject to continual increase according to the law of Eq. (8) of Section III because of the difference in the propagation velocities of the head and tail discontinuities. The third factor (3) is the actual dissipation of the wave energy $W$ (its conversion into heat) as the wave propagates.

We shall take account of all three of these factors in the Eqs. (1)-(3) and after eliminating $L$ and $W$, we shall be led to a relationship in the form of a second order differential equation connecting $y$ the "miss-distance" and $\delta$ the wave amplitude. The integral of this equation will then permit us to determine the law of decay of amplitude provided the amplitude is known at some particular miss-distance.

The energy of the $\mathrm{N}$-wave is slowly dissipated as shown in the previous sections and we have (from Eq. (10), Section II, or Eq. (8), Section III)

$$
\frac{d W}{d x} \frac{1}{W}=-\frac{\gamma+1}{2 \gamma} \frac{\delta}{L}
$$

This equation, was derived strictly for plane $\mathrm{N}$-waves. However, so long as the radius of curvature, $y \sec \theta$ of the wave front is large in comparison to the wave-length $L$, it should also be applicable to our present case with little error.

From Section I, Eq. (10.1) we have our third fundamental equation expressing the progressive increase in wave-length, $L$.

This equation also is only strictly accurate for a plane $\mathrm{N}$-wave but will be a good approximation under the same conditions as just mentioned for Eq. (2).

$$
\frac{d L}{d t}=c \frac{\gamma+1}{2 \gamma} \delta ; \quad \frac{d L}{d x}=\frac{\gamma+1}{2 \gamma} \delta .
$$

Since $y=x \cos \theta$, Eqs. (2) and (3) become, respectively,

$$
\begin{gathered}
\frac{d W}{d y} \frac{1}{W}=-\frac{\gamma+1}{2 \gamma} \frac{\delta}{L \cos \theta}, \\
\frac{d L}{d y}=\frac{\gamma+1}{2 \gamma} \frac{\delta}{\cos \theta} .
\end{gathered}
$$

Differentiating Eq. (1) logarithmically, we obtain

$$
\frac{d W}{d y} \frac{1}{W}=\frac{1}{y}+\frac{d L}{d y} \frac{1}{L}+2 \frac{d \delta}{d y} \frac{1}{\delta} .
$$

Combining this with (2.1) and (3.1)

$$
-\frac{\gamma+1}{\gamma} \frac{\delta}{L \cos \theta}=\frac{1}{y}+\frac{d \delta}{d y} \frac{1}{\delta}
$$

from which we may solve for $L$

$$
L=-\frac{\gamma+1}{\gamma} \frac{\delta y}{\cos \theta}\left[1+2 \frac{d \delta}{d y} \frac{y}{\delta}\right]^{-1} .
$$

If we make the substitution

$$
\psi=\frac{d \delta}{d y} \frac{y}{\delta}
$$

and differentiate (4.1), logarithmically we obtain

$$
\frac{d L}{d y} \frac{1}{L}=\frac{d \delta}{d y} \frac{1}{\delta}+\frac{1}{y}-2 \frac{d \psi}{d y}[1+2 \psi]^{-1} .
$$

Also, from (3.1)

$$
\frac{d L}{d y} \frac{1}{L}=\frac{1}{2 y}[1+2 \psi] .
$$

Combining these two we obtain

$$
8 \psi^{2}+10 \psi+3=4 y \frac{d \psi}{d y}
$$

which becomes, upon integration,

$$
\frac{y}{c_{1}}=\left[\frac{\psi+\frac{1}{2}}{\psi+\frac{3}{4}}\right]^{2} \text {. }
$$

We now put $y / c_{1}=y_{0}$, solve for $\psi$, and integrate once more, giving

$$
\delta=c_{2} y_{0}{ }^{-\frac{3}{2}}\left[1-y_{0}^{-\frac{1}{2}}\right]^{-\frac{1}{2}} .
$$


We may now put this expression for $\delta$ into Eq. (4.1) and obtain the dependence of wavelength on miss-distance.

$$
L=\frac{2 c_{1} c_{2}(\gamma+1)}{\gamma \cos \theta} y_{0}^{\frac{1}{4}}\left[1-y_{0}{ }^{-\frac{1}{2}}\right]^{\frac{1}{2}} .
$$

For sufficiently large miss-distances these last two equations reduce to

and

$$
\delta=(\text { const. }) y^{-\frac{3}{t}}
$$

$$
L=\text { (const.) } y^{\frac{1}{4}} \text {. }
$$

Equation (7.1) is in fairly good agreement, as we shall see, with experimental observations on .50 -caliber fire. The numerical value of the exponent is a little too small for observations on 40-mm fire however at the miss-distance we have studied, a result which may be perhaps interpreted as meaning that for this larger caliber the asymptotic rate of decay is not attained until greater miss-distances than those observed. Equation (8.1) agrees rather well with observations of the dependence of $\mathrm{N}$-wave period on miss-distance.

The constant $c_{1}$, as can readily be seen from Eq. (6), is a length, a unit peculiar to this problem for the measurement of $y$. When $y$ is measured in units, $c_{1}$, its numerical value is the variable $y_{0}$. It is clear from Eq. (7) that, as $y$ diminishes and as we approach the bullet trajectory, $c_{1}$ is a measure of the miss-distance at which, on a log-log plot, the curve of $\delta$ vs. $y$ (leparts markedly from rectilinearity. From Eq. (8) we see that when $y=c_{1},\left(y_{0}=1\right)$, the wavelength $L$ of the shock-wave has diminished to zero and it is not surprising therefore that, from Eq. (7), $\delta$ becomes infinite when $y=c_{1}$. Our present theory, which was constructed to be valid only for large miss-distances, breaks down therefore, as the miss-distance diminishes, long before $y=c_{1}$.

In order to attempt to evaluate the integration constants $c_{1}$ and $c_{2}$, the theoretical curves (7) and (8) must be compared with experimental results. Curves of the Eqs. (7) and (8) plotted on double logarithmic paper are shown in Figs. 11a and 11b. The straight lines corresponding to the asymptotic expressions, (7.1) and (8.1), are also shown. Figures 3 and 4 on the

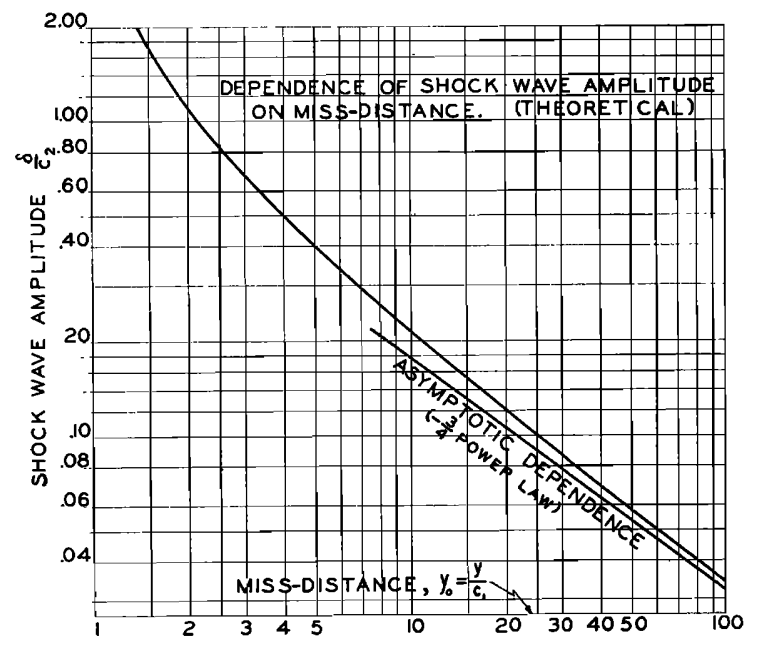

FIG.' 11a.

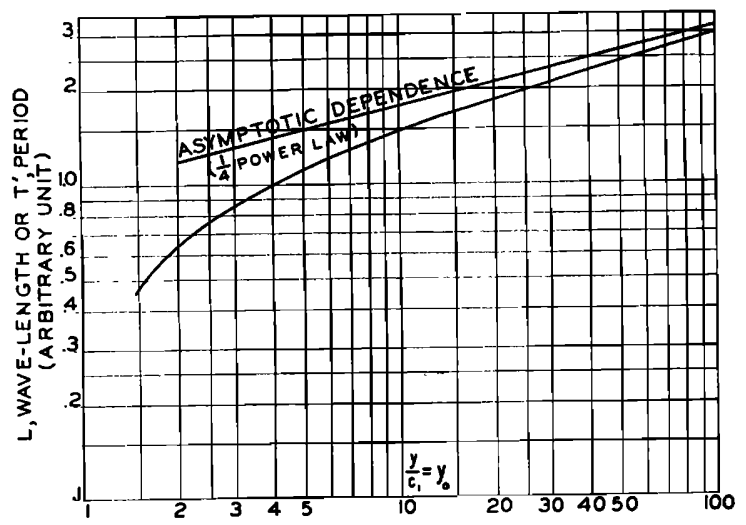

FIc. 11b. Dependence of wave-length (or period) of ballistic shock wave on miss-distance (theoretical).

other hand show the observed data. The difficulty in fitting these curves to the observed data is, unfortunately, that the scattering of the experimental points is so large as to mask any significant curvature that could lead to a determination of the constant $c_{1}$. Considering specifically Eq. (7) it may be seen that for observations at large miss-distances, the experimental points must be extremely accurate if we wish to determine the constant $c_{1}$ with any accuracy. If we use very close miss-distances on the other hand, the difficulty is even more insurmountable because of the failure there of the theory itself. Only at large distances is the assumption of complete formation of the balanced $\mathrm{N}$-wave justified. The entire argument of this section is 
based on a fully formed $\mathrm{N}$-wave which has propagated far enough from the trajectory that it may be considered to be substantially a plane wave (for the purpose of computing its energy dissipation into heat).

The easiest way to try to determine the constant $c_{1}$ is by making use of Eq. (6). This equation relates the miss-distance $y$ and the logarithmic slope, $\psi=d \log \delta / d \log y$.

Therefore we can plot observed values of $\delta=\left(p_{1}-p_{0}\right) / p_{0}$ against miss-distance on a logarithmic scale and determine the slope. Attempts to do this have met with meager success because of the inaccuracy of the observational data. For various calibers we have obtained the following results from a large quantity of field data. Each exponent $n$ listed in Table II represents the results of many hundreds of rounds fired. For 40 $\mathrm{mm}$ and .50 caliber the results of three different field trips are given. For $20 \mathrm{~mm}$ only one field trip has been held. The scattering of the value of $n$ for a given caliber is consistent with the scattering of the original data themselves and is a good index of the very real difficulty attending such measurements.

It thus appears that the variation between the different observations is so great that no reliable empirical determination of the integration constant $c_{1}$ is safe from our present data.

It is gratifying to note however that our theory predicts that the slope of the logarithmic plot of $\delta$ vs. $y$ should never be numerically less than $\frac{3}{4}$ and our field data, to within their limited accuracy, agree completely with this. One is also tempted to account for the greater observed slope in the case of $40 \mathrm{~mm}$ by concluding that $c_{1}$ is larger for this caliber than it is for .50 caliber and that our observations apply therefore to a segment of the curve of Fig. 11a somewhat

TABLE II. Logarithmic slope for $\delta$ vs. $y$ (exponent of $y$ in the approximation formula $\delta=k y^{-n}$ )

\begin{tabular}{cc}
\hline $40 \mathrm{~mm}$ & $\begin{array}{l}n=.88 \\
n=.85 \\
n=.94\end{array}$ \\
Mean of three independent determinations, $n=.89 \pm .03$ \\
\hline .50 caliber $\begin{array}{l}n=.78 \\
n=.65 \\
n=.73\end{array}$ \\
Mean of three independent determinations, $n=.72 \pm .04$ \\
\hline $20 \mathrm{~mm}$ & $n=.88$ \\
\hline
\end{tabular}

closer to $y_{0}=1$. A rough estimate of $c_{1}$ using $n=.89$ gives $c_{1}$ of the order of one yard for 40$\mathrm{mm}$ and $20-\mathrm{mm}$ shells. For .50 caliber $c_{1}$ is apparently so small as to make the factors in parenthesis in Eqs. (7) and (8) completely negligible at our working miss-distances.

The difficulty in determining the constant $c_{1}$ is just the difficulty in determining the exponent $n$ accurately. The magnitude of the variation of the experimental data may be seen from Figs. 3 and 4 which show the observed variation of peak pressure amplitude with miss-distance.

The observed wave-length of the shock wave (or to be more precise the period, which is proportional to it) varies approximately as the fourth root of the miss-distance; more accurately so as the miss-distance increases, in agreement again with the theory.

An important and surprising relation which may be obtained from Eqs. (7.1) and (8.1) relates the period of the $\mathrm{N}$-wave, the amplitude and the miss-distance and involves no arbitrary constants. We find (for large miss-distances)

$$
T^{\prime}=\frac{2(\gamma+1)}{\gamma c \cos \theta} \delta y ; \quad\left(T^{\prime} c=L\right) .
$$

This indicates that the wave-length of a ballistic shock wave, once it is completely formed, is uniquely determined at any given sufficiently large distance by specifying the amplitude of its discontinuities. The eventual wave-length is therefore apparently independent of the mechanism of generation by the bullet, being fixed by the amplitude of the wave itself rather than (for example) by the length of the bullet. Our measurements of the shock waves from all three calibers, $40 \mathrm{~mm}, 20 \mathrm{~mm}$, and .50 caliber satisfy Eq. (9) with the accuracy to which $\delta$ and $T^{\prime}$ have been determined. This is a very surprising result. Something similar to it seems to have been foreseen by Landau ${ }^{1}$ though the numerical constant he derives does not quite agree with our result.

Shock wave photographs, however, seem to indicate that for bullets of ordinary design the $H$ discontinuity comes from the nose of the bullet and the tail wave is initiated by the inrush of the air in the wake of the bullet to fill the void which its passage has created. It would be very interesting indeed, in view of Eq. (9), to examine 


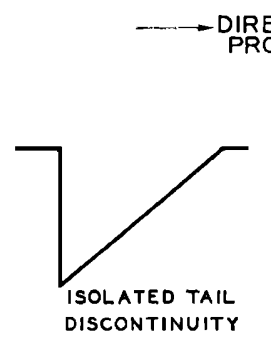

PRECTION OF

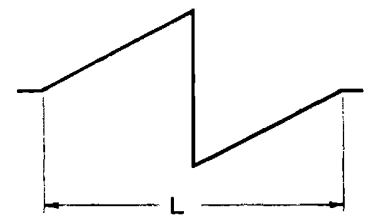

INTRAVERT N-WAVE

FIG. 12. Pressure profile of intravert $\mathrm{N}$-wave, a possible final result of propagation of an isolated tail discontinuity.

the shock wave photographs for a very long rocket or some similar very long projectile. It should be possible to make the ratio of length to diameter so great that the tail of the projectile could not possibly generate a tail wave at the correct distance behind the head wave to satisfy Eq. (9). Presumably the wave at the tail of such an elongated projectile would also bifurcate in some similar way though just how this would take place is not clear. It seems possible that the tail rarefaction from a long projectile would finally assume a balanced profile of the form shown in Fig. 12 which might be described as an intravert $\mathrm{N}$-wave. The wave-length of such a wave is invariable.

The reader may well ask why a disturbance of one sign only, such as the head wave or tail wave from a bullet, should be expected to generate spontaneously an accompanying wave of opposite sign merely by the mechanism of propagation over considerable distance. It is hardly appropriate to go deeply into this question here, but it has been pointed out by numerous authorities on theoretical acoustics ${ }^{10}$ that for the case of spherical waves where the entire disturbance is contained in an expanding spherical shell of constant thickness, the wave form eventually tends to become balanced as regards rarefaction and condensation even though initially the entire disturbance was of one sign only. Conservation of energy requires in such a case that the wave amplitude shall diminish inversely as the radial distance from the source. The wave form cannot therefore continue to consist of condensation alone for the volume of the shell increases as the square of the radius and this would violate

\footnotetext{
${ }^{20}$ See a discussion by Stokes, "On some points in the received theory of sound," Phil. Mag. 34, 52 (1849).
}

the conservation of matter as regards the excess mass in the wave. In the case of explosion waves the well-known rarefaction following in the wake of an initial condensation is an example of the case in point. In a manner of speaking, the rarefaction is formed in the process of propagation to furnish the required matter to maintain the condensation at the level imposed by the energy in the wave. It is quite possible that a similar mechanism exists for the conical ballistic shock waves under discussion. It may be that every disturbance set up by a projectile of any shape whatever, moving at supersonic velocity, must eventually bifurcate itself into an $\mathrm{N}$-wave of wave-length satisfying Eq. (9) and that the disturbances from the nose and the tail of ordinary bullets merely happen by a coincidence of the design ratios of length to diameter in ordinary bullets to cooperate in the formation of the head and tail discontinuities of one and the same $\mathrm{N}$-wave.

\section{Dependence of Amplitude and Wave-Length of Spherical Shock Waves on Miss-Distance}

In the previous section we considered the propagation of a wave from a line source, hence one that spreads out over a conical surface. We shall now adapt the results to the case of a shockwave from a point source, which spreads out in three dimensions over a spherical surface. Equations (2) and (3) of Section IV remain unchanged, the same approximations and assumptions being made here as previously. The average energy density is unchanged, but the total energy (Eq. (1)) is altered since now the wave energy is distributed over the surface of a sphere.

We have, therefore,

$$
W=4 \pi r^{2} L \frac{p_{0} \delta^{2}}{3 \gamma}
$$

in which $r=$ the distance from the source.

Furthermore,

$$
\frac{d W}{d r} \frac{1}{W}=-\frac{\gamma+1}{2 \gamma} \frac{\delta}{L}
$$

and

$$
\frac{d L}{d r}=\frac{\gamma+1}{2 \gamma} \delta,
$$


the numbers of the equations being the same as the correspondingly equivalent equations of Section IV.

By a completely similar method to that used above, we obtain

$$
\ln \frac{r}{a_{1}}=-\frac{1}{2(\psi+1)},
$$

from which, by solving for $\psi$ and integrating, we obtain the dependence of shock wave amplitude on distance

$$
\delta=\frac{C}{r\left(\ln r / a_{1}\right)^{\frac{1}{2}}}
$$

and for the dependence of wave-length on distance, we find

$$
L=\frac{\gamma+1}{\gamma} C\left(\ln r / a_{1}\right)^{\frac{1}{2}} .
$$

The constants $a_{1}$ and $C$ correspond to the constants $c_{1}$ and $c_{2}$. Eqs. (7) and (8) here are exact, not merely approximations which hold only for large distances. However, the assumptions on which they are based are such that the theory is inadequate at small distances so that the applicability of the equations is still limited to distances which are large in comparison to the wave-length.

\section{Estimation of Absolute Shock Wave Pres- sures and Energy from Measurements of $\mathrm{N}$-wave Period $T^{\prime}$}

Formula (9), Section IV, above, furnishes a convenient method for estimating the relative pressure rise (peak value) in the discontinuities of a ballistic shock wave. This formula is applicable only at large miss-distances. A formula which can be used at close miss-distances is (3.1) of the same section. We have, writing $L=T^{\prime} c, y=d=$ miss-distance, and $\gamma=1.4$ for air,

$$
\delta=\frac{p_{1}-p_{0}}{p_{0}}=\frac{7}{6} \frac{\partial T^{\prime}}{\partial d} c \cos \theta,
$$

which is our working formula. We can obtain $\partial T^{\prime} / \partial d$ from the slope of the curve of observed values of $T^{\prime}$ plotted as a function of $d$ in cartesian coordinates.

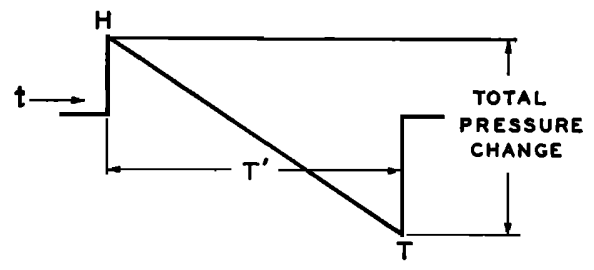

FIG. 13. Total pressure change (unbalanced $\mathrm{N}$-wave).

It must be strongly emphasized that Eq. (1) gives the pressure rise (in atmospheres) in the shock wave discontinuities only if the two ( $H$ and $T$ ) discontinuities are of equal magnitude. If they are of unequal magnitude the reader can readily check that formula (1) will give half the sum of the two magnitudes, that is to say half the total change in pressure from $H$ to $T$ indicated in the sketch below, Fig. 13.

Thus this method of estimating peak shock wave amplitudes will give values which are numerically equal to or less than the magnitude of the greater of the two discontinuity pressure jumps.

If we assume a linear decline in pressure from $H$ to $T$, that is to say a true $\mathrm{N}$-wave profile, then this method will also give the total energy in the shock wave (integrated from $H$ to $T$ ) provided the $H$ and $T$ discontinuities are of equal magnitude; but since the energy is proportional to the square of the excess pressure, $\left(p_{1}-p_{0}\right)^{2}$ if the $H$ and $T$ discontinuities are of unequal magnitude it is clear that an application of this method will give a value less than the total energy (see Section III).

A further limitation of this method comes from the fact that it is based on the formula for the excess velocity of propagation of the completely formed discontinuities in the wave form. In the earlier stages of propagation near the trajectory it is conceivable that the "mechanism of overtaking" has not had time to bring about the complete formation of the discontinuity. Thus, for example, the wave form may contain peaks which have not as yet overtaken and coalesced with the discontinuity toward which they are destined, as at $A$ or $B$ in the sketch, Fig. 14. Here again the method in question would lead to an underestimate of the pressure elevation and the energy. The method in question would give, for example, only the wave energy of an 


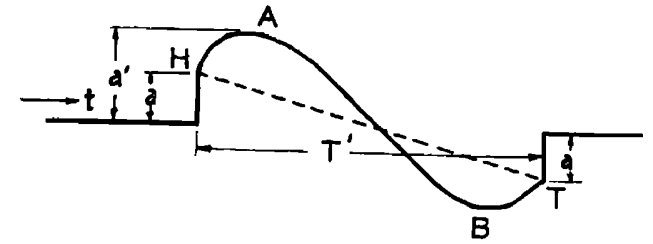

Frc. 14. Before complete formation of N-profile the $a 7^{\prime} / \partial d$ method of measuring amplitude gives only the amplitude, $a$, for an $\mathrm{N}$-wave shown by dotted line, not the peak value $a^{\prime}$.

$\mathrm{N}$-wave having the dotted line for its oblique segment rather than the energy integrated for the full line curve.

There is every reason to believe from our oscillograms as well as from theory that up to a certain point the conditions for the accuracy of the $\partial T^{\prime} / \partial d$ method are more and more closely fulfilled the farther the $\mathrm{N}$-wave is propagated from the trajectory. Not only does the profile become more and more $\mathrm{N}$-shaped by the mechanism of the higher amplitudes overtaking the lower but there is the further mechanism already alluded to which tends with propagation to make the negative and the positive sections of the wave form more and more nearly of equal amplitude. Studies of the wave forms themselves and studies of the energy associated with a shock wave calculated by the method under discussion indicate that beyond $20 \mathrm{y}^{\prime} \mathrm{cl}$. miss-distance for a shock wave from a $40-\mathrm{mm}$ shell the $\partial T^{\prime} / \partial d$ method of estimating discontinuity pressure amplitudes and wave energies is entirely reliable. On the other hand these studies indicate that for miss-distances less than $10 \mathrm{yd}$. the method is decidedly unreliable (for $40-\mathrm{mm}$ bullets) and as predicted gives pressures and energies which are too low. At sufficiently large miss-distances on the other hand the method will fail because of the limited precision with which $T^{\prime}$ can be measured when the amplitudes of the discontinuities are low relative to accidental disturbances and because of the uncertain fluctuations in $T^{\prime}$ introduced by atmospheric inhomogeneities.

It is also possible from our observations of $\partial T^{\prime} / \partial d$ to compute as a function of miss-distance the total energy associated with that part of the ballistic shock wave which came from any specified segment of the bullet trajectory on the assumption that the wave form is a balanced
$\mathrm{N}$-wave and we shall see that this leads us to a rough criterion as to the minimum miss-distance for which this method is reliable.

From Eq. (1), Section IV, and Eq. (1) above, we obtain for the energy in the shock wave per unit length of trajectory

$$
\begin{aligned}
W & =2 \pi d \cos \theta \frac{p_{0} c T^{\prime}}{3 \gamma} \delta^{2} \\
& =\frac{1 \quad 4 \gamma}{3(\gamma+1)^{2}} 2 \pi d \cos ^{3} \theta p_{0} c^{3} T^{\prime \prime}\left(\frac{\partial T^{\prime \prime}}{\partial d}\right)^{2} .
\end{aligned}
$$

This total energy should either diminish with increasing $d$ or else be constant; it should never increase since it is never replenished from any source as it is propagated from the trajectory. The velocity of a $40-\mathrm{mm}$ shell at 1000-yards range from the gun is almost exactly twice sonic velocity so that

$$
\sin \theta=0.5 \text { and } \cos \theta=.87 \text {. }
$$

If we take the velocity of sound $c=3.3 \times 10^{4} \mathrm{~cm}$ sec. ${ }^{-1}$, Formula (2) reduces to

$$
\begin{array}{r}
W=4.72 \times 10^{19} d T^{\prime}\left(\partial T^{\prime} / \partial d\right)^{2} \text { dynes (ergs per } \\
\mathrm{cm} \text { of trajectory), }
\end{array}
$$

if $T^{\prime}$ is measured in seconds and $d$ in centimeters.

Since the shock wave pressure elevation (peak value as computed from $\partial T^{\prime} / \partial d$ ) is also of interest we shall calculate this from Formula (1). In c.g.s. units this reduces numerically to

$$
p_{1}-p_{0}=3.32 \times 10^{10} \partial T^{\prime} / \partial d\left(\text { dynes } \mathrm{cm}^{-2}\right) \text {. }
$$

The results of the calculations are given in Table III. The values of $T^{\prime}$ and $\partial T^{\prime} / \partial d$ were obtained from a carefully smoothed curve plotted

TABLE III.

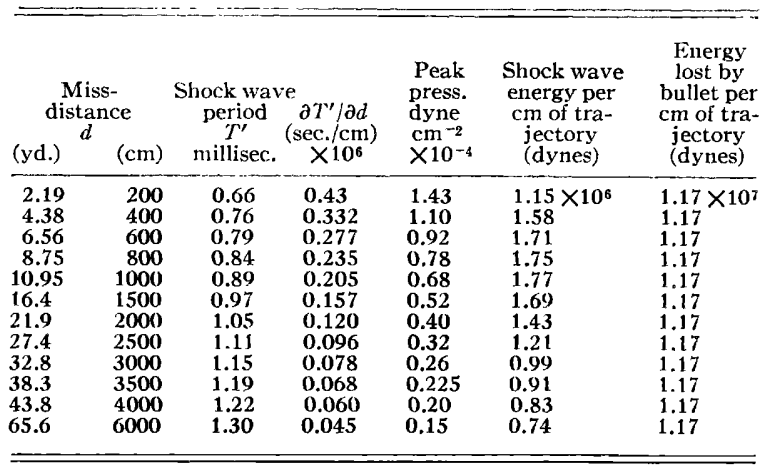




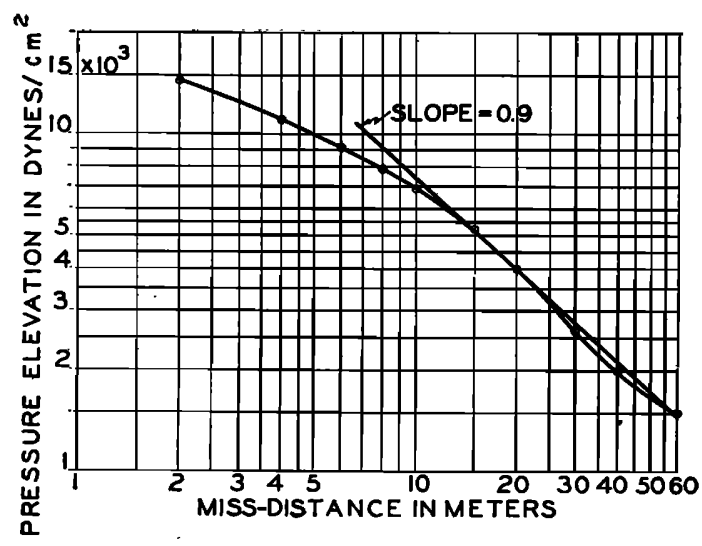

FIG. 15. Peak shock wave pressure elevation obtained by $\partial T^{\prime} / \partial d$ method and plotted as a function of the missdistance, $40-\mathrm{mm}$ bullets.

from our observed values of $T^{\prime} v s$. $d$ but too much quantitative significance should not be given to the results in consideration of the difficulty of making accurate measurements of shock wave periods and of determining the rate of change of this quantity.

In Fig. 15 we have plotted on log-log coordinates the shock wave peak pressure elevation and in Fig. 16 on semi-log coordinates the shock wave energy from unit length of trajectory (from Table III), each as a function of missdistance in meters. From 15 to 60 meters it will be noted from Fig. 15 that the peak pressure elevation as measured by the $\partial T^{\prime} / \partial d$ method falls off with increasing miss-distance in quite good agreement with our -0.9 power law as directly observed with microphones (for $40 \mathrm{~mm}$ ). At miss-distances closer than 15 meters (see Fig. 16) the shock-wave energy computed by the $\partial T^{\prime} / \partial d$ method actually increases with increasing miss-distance but thereafter decays with increasing miss-distance in a way very suggestive of a decay due to energy dissipation. Since the plot is on semi-log coordinates and the slope of this decaying curve is not constant, it is clear that the absorption coefficient of the shock wave is decreasing as the wave progresses and this is exactly what one would expect since the dissipation is localized in the discontinuities and with diminishing amplitude the thickness of these increases, with rapid resulting decrease in the rate of dissipation.

Recalling that the $\partial T^{\prime} / \partial d$ method should give values which are either equal to or less than the

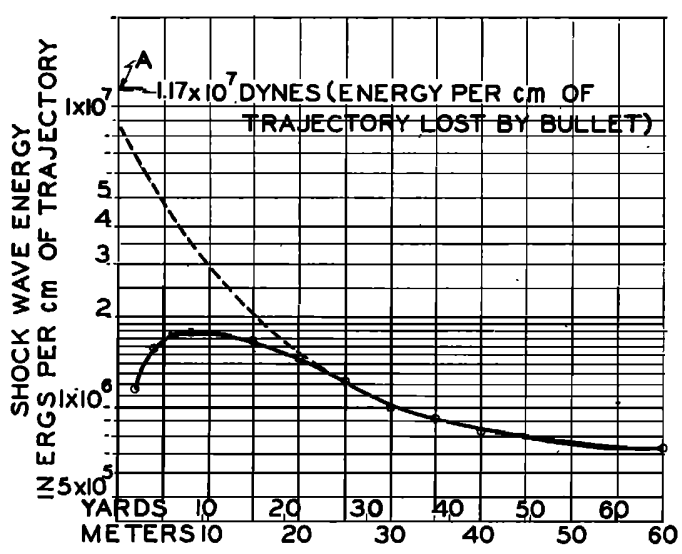

FIG. 16. Energy in the shock wave (per unit length of trajectory) obtained by $\partial T^{\prime} / \partial d$ method and plotted as a function of the miss-distance for $40-\mathrm{mm}$ bullets.

correct total energy and that the latter erroneous result is likely to occur at closer miss-distances, the curve of Fig. 16 suggest strongly that these miss-distances for which the method is unreliable extend from the trajectory out to ten or twenty meters miss.

In Fig. 16, at $A$, we have also plotted on the same logarithmic ordinate scale the energy initially available to form the shock wave, i.e., the energy lost by the bullet per unit length of trajectory. This has been directly computed from the mass of the $40-\mathrm{mm}$ bullet and its deceleration (at 1000-yard range) as taken from the ballistic tables for times of flight. This is also the figure shown in the last column of Table III.

We have taken the liberty in Fig. 16 of showing with the dotted curve how the actual shock wave energy (per unit length of bullet trajectory) may well vary with the miss-distance starting from some initial value which may be about three-quarters of the entire available energy, (the energy lost by the bullet in unit length of trajectory).

\section{Comparison of Absolute Values of Peak Shock Wave Pressure Elevations as Meas- ured (1) by the $\partial T^{\prime} / \partial d$ Method and \\ (2) by Direct Observation with Condenser Microphones}

The diaphragm of a condenser microphone facing an oncoming shock wave front should, for the weak shock waves with which we are concerned, experience a pressure change (be- 
cause of the combined effect of the reflected and incident waves) double the peak pressure elevation in the free wave (the pressure elevation as measured by the $\partial T^{\prime} / \partial d$ method).

Our condenser microphones and their associated radiofrequency oscillators are all standardized to give the same radiofrequency shift of $100 \mathrm{kc}$ when a 50 -gram weight is applied to the center of the diaphragm with a standard applicator having a standard radius of curvature at the contact point. By constructing a special pneumatic applicator for one of our microphones we have measured the air pressure (expressed in $\mathrm{mm} \mathrm{Hg}$ ) requisite to produce $100-\mathrm{kc}$ deviation and find it to be $41-\mathrm{mm} \mathrm{Hg}$. For a $40-\mathrm{mm}$ bullet at 40-yard miss-distance we olsserved a (peak) frequency shift of $8.43 \mathrm{kc}$ with our $7 \frac{1}{2}$-inch diameter spherically encased aperiodic transmitter units (provided with a similar pair of these condenser microphones) which means an applied pressure (peak value) of $3.5 \mathrm{~mm} \mathrm{Hg}$ or 0.0046 atmosphere. For the $40-\mathrm{mm}$ bullet at 40-yard miss-distance the $\partial T^{\prime} / \partial d$ method, on the other hand, gives a peak pressure in the traveling wave of 0.0022 atmosphere, corresponding to a pressure on the diaphragm (taking into account the reflected wave) of 0.0044 atmosphere. The agreement between the two methods seems to be well within the limits of experimental error. 